\title{
Triglyceride/low-Density-Lipoprotein Cholesterol (TG/LDL-C) Ratio is the Most Diagnostically Valuable Predictor for Increased Small, Dense LDL in Type 2 Diabetes Patients
}

\section{Gen Ouchi}

University of the Ryukyus Hospital

Ichiro Komiya ( $\square$ i.kom.iya@nifty.com )

Okinawa Medical Hospital https://orcid.org/0000-0002-4965-4561

Shinichiro Taira

Okinawa Rehabilitation Center Hospital

Tamio Wakugami

US Naval Hospital Okinawa

\section{Yusuke Ohya}

University of the Ryukyus Hospital

\section{Research}

Keywords: TG/LDL-C ratio, non-HDL-C, small, dense LDL, triglycerides, type 2 diabetes mellitus

Posted Date: November 8th, 2021

DOI: https://doi.org/10.21203/rs.3.rs-1037753/v1

License: (c) (i) This work is licensed under a Creative Commons Attribution 4.0 International License.

Read Full License 


\section{Abstract}

Background: Small, dense low-density lipoprotein (sd-LDL) increases in type 2 diabetes patients and causes arteriosclerosis. Non-high-density-lipoprotein cholesterol (HDL-C) is thought to be useful for predicting arteriosclerosis and sd-LDL elevation; however, there are no data about whether the triglyceride (TG)/low-density-lipoprotein cholesterol (LDL-C) ratio is a valuable predictor for sd-LDL.

Methods: One hundred and ten type 2 diabetes patients with hypertriglyceridemia were analyzed. No patients were treated with fibrates, but forty-seven patients were treated with statins. LDL-C was measured by the direct method. LDL-migration index (LDL-MI) using electrophoresis (polyacrylamide gel, PAG) was calculated, and the value $\geq 0.400$ was determined to indicate an increase in sd-LDL. Simple regression analyses were carried out between LDL-MI and lipid markers. Receiver operating characteristic curves of lipid markers for predicting high LDL-MI were applied to determine the area under the curve (AUC).

Results: LDL-MI correlated negatively with LDL-C $(P=0.0027)$ and LDL fraction $(P<0.0001)$ in PAG electrophoresis and correlated positively with TGs, non-HDL-C, TG/LDL-C ratio, TG/HDL-C ratio, and non-HDL-C/HDL-C ratio among all study patients. Similar results were obtained for patients analyzed according to statin treatment. The AUCs (95\% confidence interval) were 0.945 (0.884-1.000) for TG/LDL-C ratio and $0.614(0.463-0.765)$ for non-HDL-C in patients without statins $(P=0.0002)$. The AUCs were 0.697 (0.507-0.887) for TG/LDL-C and 0.682 (0.500-0.863) for non-HDL-C in patients treated with statins. The optimal cut-off point for TG/LDL-C ratio for increased LDL-MI was 1.1 (molar ratio) regardless of statin treatment. The sensitivity and specificity of TG/LDL-C ratio ( $90.0 \%$ and $93.9 \%)$ were higher than those of non-HDL-C (56.7\% and $78.8 \%)$ in patients without statins.

Conclusions: The TG/LDL-C ratio is a reliable surrogate lipid marker of sd-LDL and superior to non-HDL$\mathrm{C}$ in type 2 diabetes patients not treated with statins.

\section{Introduction}

The risk of cardiovascular disease (CVD) is reportedly associated with an increase in small, dense lowdensity lipoprotein (sd-LDL) levels rather than large, buoyant LDL [1]. Sd-LDL reportedly exhibits several potentially atherogenic properties, such as reduced receptor-mediated clearance, prolonged retention in circulation, greater arterial wall retention, and increased oxidation [2]. Japanese elderly men with ischemic heart disease and high sd-LDL were shown to have increased risk of CVD events over the next 5 years [3]. Higher sd-LDL occurs when both non-high-density-lipoprotein cholesterol (non-HDL-C) and triglycerides (TGs) are high [4]. Non-HDL-C, having higher atherosclerosis-inducing properties than LDL-cholesterol (LDL-C), is increased in hypertriglyceridemia [5] and associated with increased sd-LDL [6]. According to current guidelines for arteriosclerosis, non-HDL-C should be evaluated instead of LDL-C in cases such as severe hypertriglyceridemia [7]. When the non-HDL-C level is high, cholesterol-lowering therapy is 
prioritized, as in LDL cholestrolemia [7, 8]. When the TG level is $\geq 4.5 \mathrm{mmol} / \mathrm{L}$, Friedewald's formula cannot accurately evaluate LDL-C, so non-HDL-C is used as a marker instead of LDL-C.

A recent study evaluating the effect of pemafibrate on hypertriglyceridemia in type 2 diabetes reported that baseline LDL-C measured via the direct method and LDL fraction measured by polyacrylamide gel (PAG) electrophoresis decreased with increasing baseline sd-LDL [9]. Pemafibrate, a selective peroxisome proliferator-activated receptor alpha modulator, allows control of serum TG levels and sd-LDL, which were previously inadequate with conventional treatment $[10,11]$. In more than half of type 2 diabetes patients with hypertriglyceridemia, baseline LDL-C is relatively lower than after pemafibrate administration [9]. Higher baseline TG level also appears to be involved in the baseline sd-LDL increase and post-dose LDL-C increase. It was suggested that decreasing large, buoyant LDL reflects the bulk of the measured LDL-C in untreated type 2 diabetes patients with hypertriglyceridemia.

When non-HDL-C was used as a lipid marker, no change in LDL composition was observed; that is, if the increase in TGs and decrease in LDL (mainly large, buoyant LDL) occur synchronously, non-HDL-C does not change markedly. This was shown in our previous study examining the effect of pemafibrate [9]. Diabetes patients with high TGs but normal or low LDL-C may have higher sd-LDL. We hypothesized that the TG/LDL-C ratio would be useful for predicting higher sd-LDL or apolipoprotein-B (apoB)-containing lipoproteins as an alternative to non-HDL-C. The present study clarified whether the TG/LDL-C ratio is more valuable than non-HDL-C or other lipid markers in predicting sd-LDL level in type 2 diabetes patients treated with or without statins.

\section{Materials And Methods}

\section{Patients and study procedures}

Subjects in this retrospective study were outpatients with type 2 diabetes visiting the Medical Plaza Daido Central. A total of 130 patients with type 2 diabetes with hypertriglyceridemia were enrolled in this study after excluding heavy drinkers and patients with an estimated glomerular filtration rate (eGFR) $<45$ $\mathrm{mL} / \mathrm{min}$. Twenty patients treated with conventional fibrates, ezetimibe, or sodium-glucose cotransporter 2 inhibitors were also excluded. The final study group consisted of 110 patients, including 47 patients treated with statins and 63 not treated with statins. Because TG levels tend to fluctuate under the influence of diet [12], it was confirmed that fasting TGs were $\geq 1.7 \mathrm{mmol} / \mathrm{L}(150 \mathrm{mg} / \mathrm{dL})$ by repeated measurements in the 110 diabetes patients enrolled in the study. The average of 2 or 3 measurements was used as the baseline value. Blood samples were collected after 9-12 $\mathrm{h}$ of fasting.

Subjects with a TG level of $\geq 11.3 \mathrm{mmol} / \mathrm{L}(1,000 \mathrm{mg} / \mathrm{dL})$ were excluded from this study due to reduced reliability of LDL-C levels measured via the direct method. Lipoprotein electrophoresis (PAG) was conducted in all patients. PAG electrophoresis revealed 4 lipoprotein fractions (HDL, LDL, midband, and very-low-density lipoprotein [VLDL]). The LDL-migration index (LDL-MI) was calculated from the pattern of PAG electrophoresis according to a previous report $[13,14]$; that is, the PAG electrophoretic distance 
between the LDL and VLDL fractions was divided by that between the HDL and VLDL fractions. When this value was $\geq 0.400$, it was determined to indicate an increase in sd-LDL $[13,14]$. LDL-C was measured via the direct homogenized method $[15,16]$.

\section{Statistical analysis}

Data are shown as the mean \pm SD (normal distribution), median (interquartile range [IQR]) (nonparametric distribution), or percentage. Results of TG and TG-related variable analyses are shown as the median (25\%-75\% quartiles, IQR) due to non-parametric distribution. Comparisons between groups were made using $t$-test, Mann-Whitney $U$ test, or the $\chi^{2}$ test. A simple regression analysis using the least squares method was applied for continuous variables (TGs, LDL-C, non-HDL-C, TG/LDL-C ratio, TG/HDL-C ratio, LDL/HDL-C ratio, non-HDL-C/HDL-C ratio, LDL, midband, and VLDL fractions in PAG) as explanatory variables and with LDL-MI as a response variable to determine the regression coefficient $(r)$, standard error (SE), 95\% confidence interval (Cl), and standardized $R^{2}$ values. LDL-MI $\geq 0.400$ was predictive of higher sd-LDL level. A receiver operating characteristic (ROC) curve was generated to evaluate the discriminatory ability of the variables for higher LDL-MI, and the area under the curve (AUC) with its $95 \%$ $\mathrm{Cl}$ was calculated. To determine the optimal cut-off points of LDL-C, TGs, non-HDL-C, LDL and midband fractions in PAG, TG/LDL-C ratio, TG/HDL-C ratio, LDL-C/HDL-C ratio, and non-HDL-C/HDL-C ratio, the square root of $\left([1-\text { sensitivity }]^{2}+[1-\text { specificity }]^{2}\right)$ was calculated, which represented the point on the ROC curve with the shortest distance from the upper left corner.

JMP for Windows software, version 12 (SAS Institute Japan; Tokyo, Japan), was used for statistical analyses. ROC analyses were performed using EZR (Saitama Medical Center, Jichi Medical University, Saitama, Japan), a graphical user interface for R (The R Foundation for Statistical Computing, Vienna, Austria) [17]. $P$ values of $<0.05$ were considered statistically significant.

\section{Results}

\section{Comparison of clinical parameters in type 2 diabetes patients with hypertriglyceridemia}

The clinical parameters of 110 type 2 diabetes patients are shown in Table 1. The proportion of males was high. Mean body mass index was 26.7 (SD 3.9) $\mathrm{kg} / \mathrm{m}^{2}$. The average glycated hemoglobin was 7.3 (1.4)\%, and the average eGFR was $72.4(18.8) \mathrm{mL} / \mathrm{min} / 1.73 \mathrm{~m}^{2}$. Hypertension and CVD/stroke complications were reported for $67.3 \%$ and $19.1 \%$ of patients, respectively. Comparing patients with and without statin treatment, the mean age was higher in patients treated with statins (66.6 [SD 9.8] years) than in those not treated with statins (59.4 [13.6] years) $(P=0.0027, t$-test), and the average eGFR was lower in patients treated with statins $\left(66.6[13.4] \mathrm{mL} / \mathrm{min} / 1.73 \mathrm{~m}^{2}\right)$ than in those not treated with statins $\left(76.7[21.1] \mathrm{mL} / \mathrm{min} / 1.73 \mathrm{~m}^{2}\right)(P=0.0051)$. However, there were no significant differences in other clinical parameters between the two groups. With regard to lipid markers, patients treated with statins were characterized by lower TG, higher HDL-C, and lower non-HDL-C levels compared with patients not treated 
with statins. Patients treated with statins were characterized by lower TG/HDL-C and non-HDL-C/HDL-C ratios compared with patients not treated with statins (Table 1). 
Table 1

Characteristics of type 2 diabetes patients with hypertriglyceridemia

\begin{tabular}{|c|c|c|c|c|}
\hline Variables & Total & With statins & Without statins & $P *$ \\
\hline Number (\%) & 110 & 47 & 63 & \\
\hline Age, mean $\pm S D$, years & $62.5 \pm 12.6$ & $66.6 \pm 9.8$ & $59.4 \pm 13.6$ & 0.0027 \\
\hline Men: Women & $70: 40$ & $26: 21$ & $44: 19$ & 0.1173 \\
\hline $\mathrm{BMI}$, mean $\pm \mathrm{SD}, \mathrm{kg} / \mathrm{m}^{2}$ & $26.7 \pm 3.9$ & $26.8 \pm 4.3$ & $26.6 \pm 3.6$ & 0.7968 \\
\hline $\mathrm{HbA} 1 \mathrm{c}$, mean $\pm \mathrm{SD}, \%$ & $7.3 \pm 1.4$ & $7.4 \pm 1.4$ & $7.2 \pm 1.5$ & 0.6683 \\
\hline $\begin{array}{l}\text { eGFR, mean } \pm \mathrm{SD}, \mathrm{mL} \\
/ \mathrm{min} / 1.73 \mathrm{~m}^{2}\end{array}$ & $72.4 \pm 18.8$ & $66.6 \pm 13.4$ & $76.7 \pm 21.1$ & 0.0051 \\
\hline Complications & $74(67.3)$ & $34(72.3)$ & $40(63.5)$ & 0.3279 \\
\hline Hypertension, no. (\%) & $21(19.1)$ & 9 (19.2) & $12(19.1)$ & 0.9893 \\
\hline \multicolumn{5}{|l|}{ CVD/stroke, no. (\%) } \\
\hline Treatment for diabetes & $60(54.5)$ & $30(63.8)$ & $30(47.6)$ & \multirow[t]{4}{*}{0.2835} \\
\hline OHA, no. (\%) & $16(14.5)$ & $7(15.0)$ & $9(14.3)$ & \\
\hline Insulin + OHA, no. (\%) & $4(3.6)$ & $1(2.1)$ & $3(4.8)$ & \\
\hline \multicolumn{4}{|l|}{ Insulin, no (\%) } & \\
\hline TG, median (IQR), mmol/L & $3.1(2.4-4.2)$ & $2.9(2.2-3.5)$ & $3.3(2.5-5.3)$ & 0.0167 \\
\hline $\mathrm{LDL}-\mathrm{C}$, mean $\pm \mathrm{SD}, \mathrm{mmol} / \mathrm{L}$ & $2.9 \pm 0.9$ & $2.9 \pm 0.7$ & $2.9 \pm 1.0$ & 0.9221 \\
\hline $\mathrm{HDL}-\mathrm{C}$, mean $\pm \mathrm{SD}, \mathrm{mmol} / \mathrm{L}$ & $1.2 \pm 0.3$ & $1.3 \pm 0.3$ & $1.2 \pm 0.3$ & 0.0150 \\
\hline $\begin{array}{l}\text { Non-HDL-C, mean } \pm S D \text {, } \\
\mathrm{mmol} / \mathrm{L}\end{array}$ & $4.6 \pm 1.0$ & $4.3 \pm 0.8$ & $4.8 \pm 1.1$ & 0.0122 \\
\hline TG/LDL-C ratio, median (IQR) & $1.0(0.8-1.7)$ & $0.9(0.7-1.3)$ & $1.1(0.8-2.2)$ & 0.0803 \\
\hline TG/HDL-C ratio, median (IQR) & $2.5(1.8-3.0)$ & $2.3(1.5-3.1)$ & $2.7(1.9-5.4)$ & 0.0169 \\
\hline LDL-C/HDL-C ratio, mean \pm SD & $2.5 \pm 0.9$ & $2.3 \pm 0.8$ & $2.6 \pm 0.9$ & 0.1050 \\
\hline $\begin{array}{l}\text { Non-HDL-C/HDL-C ratio, mean } \\
\pm \text { SD }\end{array}$ & $4.1 \pm 1.8$ & $3.5 \pm 1.3$ & $4.5 \pm 2.0$ & 0.0039 \\
\hline
\end{tabular}

* $P$, t-test or Mann-Whitney $\mathrm{U}$ test between patients with and without statins. BMI, body mass index; $\mathrm{HbA1c}$, glycated hemoglobin; eGFR, estimated glomerular filtration rate; CVD, cardiovascular diseases; $\mathrm{OHA}$, oral hypoglycemic agents; TG, triglycerides; LDL-C, low-density lipoprotein cholesterol; Non-HDL-C, non-high-density lipoprotein cholesterol; HDL-C, high-density lipoprotein cholesterol; LDL-MI, LDL migration index. 


\begin{tabular}{|lllll|}
\hline Variables & Total & With statins & Without statins & $P^{*}$ \\
\hline Lipoprotein fraction & $20.7 \pm 5.6$ & $21.4 \pm 4.5$ & $20.2 \pm 6.4$ & 0.2620 \\
(PAG electrophoresis) & $39.7 \pm 12.7$ & $41.7 \pm 10.0$ & $38.2 \pm 14.3$ & 0.1544 \\
HDL, mean \pm SD, \% & $17.6 \pm 8.5$ & $17.7 \pm 7.8$ & $17.5 \pm 9.0$ & 0.9419 \\
LDL, mean \pm SD, \% & $20.1 \pm 8.4$ & $19.1 \pm 6.5$ & $24.1 \pm 9.1$ & 0.0023 \\
Midband, mean \pm SD, \% & $0.393(0.353-$ & $0.381(0.350-$ & $0.398(0.355-$ & 0.0781 \\
VLDL, mean \pm SD, \% & $0.420)$ & $0.404)$ & $0.426)$ & \\
LDL-MI, median (IQR) & & & & \\
\hline $\begin{array}{l}\text { * P, t-test or Mann-Whitney U test between patients with and without statins. BMI, body mass index; } \\
\text { HbA1C, glycated hemoglobin; eGFR, estimated glomerular filtration rate; CVD, cardiovascular diseases; } \\
\text { OHA, oral hypoglycemic agents; TG, triglycerides; LDL-C, low-density lipoprotein cholesterol; }\end{array}$ \\
$\begin{array}{l}\text { Non-HDL-C, non-high-density lipoprotein cholesterol; HDL-C, high-density lipoprotein cholesterol; } \\
\text { LDL-MI, LDL migration index. }\end{array}$ \\
\hline
\end{tabular}

Table 1 also shows the results of lipoprotein fraction analyses and LDL-MI in PAG electrophoresis. PAG electrophoresis revealed 4 lipoprotein fractions (HDL, LDL, midband, and VLDL), but there were cases in which the midband fraction was not present. The VLDL fraction was lower in patients treated with statins than in those not treated with statins (19.3 [SD 6.5]\% vs. $24.1[9.1] \%, P=0.0023)$. There was no statistical difference in LDL fraction between patients treated with statins and those not treated with statins (41.7 $[10.0] \%$ vs. $38.3[14.3] \%, P=0.1544)$. The LDL-MI in patients treated with statins was 0.381 (IQR $0.350-$ 0.404), which was lower than that in patients not treated with statins $(0.498$ [0.355-0.426), but the difference was not statistically significant $(P=0.0781)$.

\section{Simple regression analyses between lipid markers and LDL- MI}

In simple regression correlation analyses of the 110 patients, LDL-MI levels correlated with 10 lipid markers, except LDL-C/HDL-C ratio (Table 2). An inverse correlation was observed between LDL-MI and LDL fraction (PAG) $\left(R^{2}=0.2396, P<0.0001\right.$, least squares) and LDL-MI and LDL-C $\left(R^{2}=0.0804, P=\right.$ 0.0027). Positive correlations were observed between LDL-MI and the other 7 lipid markers. When patients were analyzed based on statin treatment status, an inverse correlation was observed between LDL-MI and LDL fraction in patients treated with statins $\left(R^{2}=0.1351, P=0.0113\right)$ and in those not treated with statins $\left(R^{2}=0.2668, P<0.0001\right)$. Results similar to those obtained for all study patients were observed when examining the relationship between the other 9 lipid markers and LDL-MI according to statin treatment status. 
Table 2

Simple regression analysis between LDL-MI and lipid markers in 110 type 2 diabetes patients with hypertriglyceridemia

\begin{tabular}{|c|c|c|c|c|c|c|}
\hline \multirow[t]{2}{*}{ Variables } & \multirow[t]{2}{*}{$\mathbf{r}$} & \multirow[t]{2}{*}{ SE } & \multicolumn{2}{|l|}{$95 \% \mathrm{Cl}$} & \multirow[t]{2}{*}{$P^{*}$} & \multirow[t]{2}{*}{ Adjusted $\mathrm{R}^{2}$} \\
\hline & & & Lower & Upper & & \\
\hline \multicolumn{7}{|l|}{ Total } \\
\hline $\mathrm{TG}, \mathrm{mmol} / \mathrm{L}$ & 0.0188 & 0.0021 & 0.0147 & 0.0229 & $<0.0001$ & 0.4429 \\
\hline LDL-C, mmol/L & -0.0183 & 0.0060 & -0.0301 & -0.0065 & 0.0027 & 0.0804 \\
\hline Non-HDL-C, mmol/L & 0.0207 & 0.0050 & 0.0108 & 0.0307 & $<0.0001$ & 0.1359 \\
\hline TG/LDL-C ratio & 0.0222 & 0.0030 & 0.0161 & 0.0282 & $<0.0001$ & 0.3299 \\
\hline TG/HDL-C ratio & 0.0109 & 0.0015 & 0.0080 & 0.0139 & $<0.0001$ & 0.3390 \\
\hline LDL-C/HDL-C ratio & 0.0019 & 0.0063 & -0.0105 & 0.0144 & 0.7572 & 0.0009 \\
\hline Non-HDL-C/HDL-C ratio & 0.0150 & 0.0027 & 0.0097 & 0.0203 & $<0.0001$ & 0.2252 \\
\hline LDL (PAG), \% & -0.0021 & 0.0004 & -0.0029 & -0.0014 & $<0.0001$ & 0.2396 \\
\hline Midband (PAG), \% & 0.0031 & 0.0006 & 0.0020 & 0.0042 & $<0.0001$ & 0.2308 \\
\hline VLDL (PAG), \% & 0.0024 & 0.0006 & 0.0012 & 0.0036 & 0.0001 & 0.1309 \\
\hline \multicolumn{7}{|l|}{ With statins } \\
\hline TG, $\mathrm{mmol} / \mathrm{L}$ & 0.0156 & 0.0033 & 0.0089 & 0.0222 & $<0.0001$ & 0.3314 \\
\hline LDL-C, mmol/L & -0.0077 & 0.0094 & -0.0266 & 0.0113 & 0.4203 & 0.0149 \\
\hline Non-HDL-C, mmol/L & 0.0227 & 0.0072 & 0.0083 & 0.0372 & 0.0027 & 0.1826 \\
\hline TG/LDL-C ratio & 0.0167 & 0.0045 & 0.0077 & 0.0258 & 0.0006 & 0.2349 \\
\hline TG/HDL-C ratio & 0.0113 & 0.0027 & 0.0059 & 0.0167 & 0.0001 & 0.2825 \\
\hline LDL-C/HDL-C ratio & 0.0049 & 0.0085 & -0.0122 & 0.0220 & 0.5687 & 0.0073 \\
\hline Non-HDL-C/HDL-C ratio & 0.0152 & 0.0045 & 0.0061 & 0.0243 & 0.0015 & 0.2018 \\
\hline LDL (PAG), \% & -0.0016 & 0.0006 & -0.0028 & -0.0004 & 0.0110 & 0.1351 \\
\hline Midband (PAG), \% & 0.0033 & 0.0007 & 0.0020 & 0.0046 & $<0.0001$ & 0.3588 \\
\hline VLDL (PAG), \% & 0.0007 & 0.0010 & -0.0013 & 0.0026 & 0.4999 & 0.0102 \\
\hline
\end{tabular}

r: regression coefficient. SE: standard error. 95\% Cl: 95\% confidence interval. *, Statistical analysis by least squares method. TG, triglycerides; LDL-C, low-density lipoprotein cholesterol; Non-HDL-C, non-high-density lipoprotein cholesterol; HDL-C, high-density lipoprotein cholesterol; HDL, highdensity lipoprotein; LDL, low-density lipoprotein; VLDL, very low-density lipoprotein; PAG, polyacrylamide gel. 


\begin{tabular}{|c|c|c|c|c|c|c|}
\hline \multirow[t]{2}{*}{ Variables } & \multirow[t]{2}{*}{$\mathbf{r}$} & \multirow[t]{2}{*}{ SE } & \multicolumn{2}{|l|}{$95 \% \mathrm{Cl}$} & \multirow[t]{2}{*}{$P^{*}$} & \multirow{2}{*}{ Adjusted $\mathrm{R}^{2}$} \\
\hline & & & Lower & Upper & & \\
\hline \multicolumn{7}{|l|}{ Without statins } \\
\hline $\mathrm{TG}, \mathrm{mmol} / \mathrm{L}$ & 0.0199 & 0.0028 & 0.0145 & 0.0254 & $<0.0001$ & 0.4520 \\
\hline LDL-C, mmol/L & -0.0222 & 0.0076 & -0.0374 & -0.0070 & 0.0050 & 0.1222 \\
\hline Non-HDL-C, mmol/L & 0.0181 & 0.0071 & 0.0038 & 0.0323 & 0.0139 & 0.0953 \\
\hline TG/LDL-C ratio & 0.0240 & 0.0042 & 0.0157 & 0.0323 & $<0.0001$ & 0.3542 \\
\hline TG/HDL-C ratio & 0.0106 & 0.0019 & 0.0068 & 0.0145 & $<0.0001$ & 0.3328 \\
\hline LDL-C/HDL-C ratio & 0.0022 & 0.0088 & -0.0198 & 0.0153 & 0.7994 & 0.0011 \\
\hline Non-HDL-C/HDL-C ratio & 0.0143 & 0.0036 & 0.0071 & 0.0216 & 0.0002 & 0.2033 \\
\hline LDL (PAG), \% & -0.0022 & 0.0005 & -0.0032 & -0.0013 & $<0.0001$ & 0.2668 \\
\hline Midband (PAG), \% & 0.0031 & 0.0008 & 0.0015 & 0.0047 & 0.0003 & 0.1975 \\
\hline VLDL (PAG), \% & 0.0028 & 0.0008 & 0.0012 & 0.0044 & 0.0007 & 0.1713 \\
\hline
\end{tabular}

\section{AUC and cut-off points for non-HDL-C, TG/LDL-C ratio, and other lipid markers for LDL-MI determination}

A receiver operating characteristic (ROC) curve was generated to evaluate the discriminatory ability of 9 lipid markers for LDL-MI, and the AUC was then calculated (Table 3). Among all patients with TG $>1.7$ $\mathrm{mmol} / \mathrm{L}$ analyzed using LDL-MI $\geq 0.400$ as the gold standard, the AUC for TG/LDL-C ratio (0.865 [0.7860.945]) was the highest among the 9 lipid markers examined. The optimal cut-off points for TG/LDL-C ratio, non-HDL-C, TG/HDL-C ratio, and non-HDL-C/HDL-C ratio for increased LDL-MI were 1.1, 4.9 $\mathrm{mmol} / \mathrm{L}, 2.6$, and 3.8, respectively. The sensitivity of the TG/LDL-C ratio for predicting higher sd-LDL was calculated as $86.4 \%$, with a specificity of $86.4 \%$. Both the sensitivity and specificity of the TG/LDL-C ratio were higher than those of non-HDL-C (52.3\% and $81.8 \%$, respectively), TG/HDL-C ratio $(75.0 \%$ and $75.8 \%$, respectively), and non-HDL-C/HDL-C ratio ( $68.2 \%$ and $68.2 \%$, respectively). Figure $1 \mathrm{~A}$ shows ROC curves for TG/LDL-C ratio and non-HDL-C among all study patients, and there was significant difference in AUC between the TG/LDL-C ratio and non-HDL-C $(P=0.0035)$. 
Table 3

Comparison of AUC, sensitivity, and specificity for each marker in predicting higher LDL-MI

\begin{tabular}{|c|c|c|c|c|}
\hline Variables & $\operatorname{AUC}(95 \% \mathrm{Cl})$ & Sensitivity & Specificity & Cut-off point \\
\hline \multicolumn{5}{|l|}{ Total $(n=110)$} \\
\hline TG & $0.854(0.783-0.926)$ & $79.5 \%$ & $72.7 \%$ & $3.1 \mathrm{mmol} / \mathrm{L}$ \\
\hline LDL-C† & $0.658(0.543-0.773)$ & $61.4 \%$ & $71.2 \%$ & $2.8 \mathrm{mmol} / \mathrm{L}$ \\
\hline Non-HDL-C & $0.643(0.528-0.758)$ & $52.3 \%$ & $81.8 \%$ & $4.9 \mathrm{mmol} / \mathrm{L}$ \\
\hline LDL fraction $(P A G) \dagger$ & $0.766(0.679-0.854)$ & $70.5 \%$ & $66.7 \%$ & $41 \%$ \\
\hline Midband fraction (PAG) & $0.738(0.644-0.832)$ & $75.0 \%$ & $65.2 \%$ & $17 \%$ \\
\hline TG/LDL-C ratio & $0.865(0.786-0.945)$ & $86.4 \%$ & $86.4 \%$ & 1.1 \\
\hline TG/HDL-C ratio & $0.844(0.772-0.917)$ & $75.0 \%$ & $75.8 \%$ & 2.6 \\
\hline LDL-C/HDL-C ratio & $0.516(0.398-0.635)$ & $47.7 \%$ & $62.1 \%$ & 2.2 \\
\hline Non-HDL-C/HDL-C ratio & $0.717(0.615-0.818)$ & $68.2 \%$ & $68.2 \%$ & 3.8 \\
\hline \multicolumn{5}{|l|}{ With statins ( $n=47$ ) } \\
\hline TG & $0.755(0.604-0.907)$ & $71.4 \%$ & $72.7 \%$ & $3.1 \mathrm{mmol} / \mathrm{L}$ \\
\hline LDL-C + & $0.466(0.262-0.671)$ & $57.1 \%$ & $57.6 \%$ & $2.8 \mathrm{mmol} / \mathrm{L}$ \\
\hline Non-HDL-C & $0.682(0.500-0.863)$ & $64.3 \%$ & $69.7 \%$ & $4.5 \mathrm{mmol} / \mathrm{L}$ \\
\hline LDL fraction $(P A G) \dagger$ & $0.656(0.491-0.821)$ & $64.3 \%$ & $66.7 \%$ & $41 \%$ \\
\hline Midband fraction (PAG) & $0.824(0.695-0.952)$ & $78.6 \%$ & $75.8 \%$ & $21 \%$ \\
\hline TG/LDL-C ratio & $0.697(0.507-0.887)$ & $78.6 \%$ & $78.3 \%$ & 1.1 \\
\hline TG/HDL-C ratio & $0.747(0.602-0.892)$ & $78.6 \%$ & $63.6 \%$ & 2.3 \\
\hline LDL-C/HDL-C ratio & $0.623(0.448-0.799)$ & $64.3 \%$ & $63.6 \%$ & 2.4 \\
\hline Non-HDL-C/HDL-C ratio & $0.691(0.527-0.854)$ & $64.3 \%$ & $69.7 \%$ & 3.5 \\
\hline \multicolumn{5}{|l|}{ Without statins $(n=63)$} \\
\hline TG & $0.910(0.839-0.981)$ & $80.0 \%$ & $87.9 \%$ & $3.3 \mathrm{mmol} / \mathrm{L}$ \\
\hline LDL-C† & $0.760(0.630-0.889)$ & $63.3 \%$ & $90.9 \%$ & $2.6 \mathrm{mmol} / \mathrm{L}$ \\
\hline Non-HDL-C & $0.614(0.463-0.765)$ & $56.7 \%$ & $78.8 \%$ & $5.0 \mathrm{mmol} / \mathrm{L}$ \\
\hline LDL fraction $(P A G) \dagger$ & $0.838(0.735-0.929)$ & $63.3 \%$ & $90.9 \%$ & $35 \%$ \\
\hline
\end{tabular}

$\mathrm{Cl}$, confidence interval; TG, triglycerides; LDL-C, low-density lipoprotein cholesterol; Non-HDL-C, non-high-density lipoprotein cholesterol; HDL-C, high-density lipoprotein cholesterol. + , the lipid markers decrease with the increase of LDL-MI. 


\begin{tabular}{|lllll|}
\hline Variables & AUC $(95 \%$ Cl) & Sensitivity & Specificity & Cut-off point \\
\hline Midband fraction (PAG) & $0.701(0.571-0.831)$ & $70.0 \%$ & $69.7 \%$ & $17 \%$ \\
\hline TG/LDL-C ratio & $0.945(0.884-1.000)$ & $90.0 \%$ & $93.9 \%$ & 1.1 \\
\hline TG/HDL-C ratio & $0.892(0.815-0.969)$ & $80.0 \%$ & $78.8 \%$ & 2.7 \\
\hline LDL-C/HDL-C ratio & $0.591(0.435-0.747)$ & $53.3 \%$ & $81.8 \%$ & 2.2 \\
\hline Non-HDL-C/HDL-C ratio & $0.724(0.590-0.859)$ & $66.7 \%$ & $81.8 \%$ & 4.3 \\
\hline $\begin{array}{l}\text { Cl, confidence interval; TG, triglycerides; LDL-C, low-density lipoprotein cholesterol; Non-HDL-C, } \\
\text { non-high-density lipoprotein cholesterol; HDL-C, high-density lipoprotein cholesterol. +, the lipid } \\
\text { markers decrease with the increase of LDL-MI. }\end{array}$ & \multicolumn{4}{l}{} \\
\hline
\end{tabular}

Among patients analyzed after grouping by statin use, the AUC for TG/LDL-C ratio (0.945 [0.884-1.000]) in patients not treated with statins was higher than the AUCs for the other lipid markers in patients with or without statin treatment. Figure 1B shows ROC curves for TG/LDL-C ratio and non-HDL-C in patients not treated with statins, and there was significant difference in AUC between TG/LDL-C ratio and non-HDL-C $(P=0.0002)$. In patients not treated with statins, the optimal cut-off points for TG/LDL-C ratio, non-HDLC, TG/HDL-C ratio, and non-HDL-C/HDL-C ratio for increased LDL-MI were 1.1, $5.0 \mathrm{mmol} / \mathrm{L}, 2.7$, and 4.3, respectively. The sensitivity of the TG/LDL-C ratio for predicting higher sd-LDL was calculated as $90.0 \%$, with a specificity of $93.9 \%$. Both the sensitivity and specificity of the TG/LDL-C ratio were higher than those of non-HDL-C (56.7\% and $78.8 \%$, respectively), TG/HDL-C ratio ( $80.0 \%$ and $78.8 \%$, respectively), and non-HDL-C/HDL-C ratio ( $66.7 \%$ and $81.8 \%$, respectively). In patients treated with statins, the optimal cut-off points for TG/LDL-C ratio, non-HDL-C, TG/HDL-C ratio, and non-HDL-C/HDL-C ratio for increased LDL-MI were 1.1, $4.5 \mathrm{mmol} / \mathrm{L}, 2.3$, and 3.5, respectively. The sensitivity of TG/LDL-C ratio (78.6\%) was the same as those of midband and TG/HDL-C ratio but higher than that of either non-HDL-C $(64.3 \%)$ or non-HDL-C/HDL-C ratio (64.3\%). There was no difference in the cut-off point for the TG/LDL-C ratio for high LDL-MI between patients with and without statin treatment. Although the cut-off points for other lipid markers for higher LDL-MI varied widely depending on statin treatment status, the TG/LDL-C ratio remained constant at approximately 1.1 .

\section{Discussion}

The present study demonstrated for the first time the usefulness of the TG/LDL-C ratio as a predictive marker for higher sd-LDL in type 2 diabetes patients with hypertriglyceridemia. In statin-free patients, the assumed cut-off point for TG/LDL-C ratio was 1.1, and the sensitivity and specificity of the ratio as a predictive marker for higher sd-LDL surpass those of non-HDL-C or other lipid markers. TG/LDL-C ratio is the first formula proposed and considered suitable for evaluation of sd-LDL and TG-rich lipoproteins. TGs were positively correlated with LDL-MI, and LDL fraction (PAG) and LDL-C were negatively correlated with LDL-MI. The TG/LDL-C ratio more reliably predicts an increase in LDL-MI (sd-LDL). Even if LDL-C is low or within the normal range, it is possible to predict high values of sd-LDL by calculating the relative ratio with TGs. The reciprocal of this formula, LDL-C/TG ratio, was reported by Yoshida et al. and suggested as 
being related to sd-LDL [18]. In the present study, TG level was used as the numerator for the purpose of emphasizing the existence of TG-rich apoB-containing lipoproteins.

In a study involving 994 non-diabetic patients with TGs of $\leq 4.5 \mathrm{mmol} / \mathrm{L}(400 \mathrm{mg} / \mathrm{dL})$, the AUC of nonHDL-C was 0.871 (0.840-0.901), the sensitivity was $78.8 \%$, specificity $79.8 \%$, and positive predictive value $54.9 \%$ for predicting high sd-LDL ( $\geq 46 \mathrm{mg} / \mathrm{dL}$ via direct assay) [19]. In that study, LDL-C was calculated using Friedewald's formula. A simple comparison between the previous and present studies shows that the TG/LDL-C ratio of the present study is superior in terms of AUC, sensitivity, and specificity versus non-HDL-C of the previous study for the prediction of high sd-LDL.

Specific clinical data collected in routine clinical practice can be combined with other related data to increase clinical usefulness for the diagnosis or estimation of various diseases [20]. The routine lipid panel consists of LDL-C, HDL-C, TGs, and total cholesterol. Several additional parameters, such as nonHDL-C (total cholesterol minus HDL-C), LDL-C/HDL-C ratio [21], non-HDL-C/HDL-C ratio [22], and TG/HDL-C ratio [23], are emerging as valuable adjuncts to the standard panel. Non-HDL-C is used to evaluate apoB-containing lipoproteins and sd-LDL [24]. In particular, increased non-HDL-C concentration is reportedly associated with residual risk for CVD and has been adopted as a guideline for lipid management [7]. In general, when non-HDL-C increases, the cholesterol contained in TG-rich lipoprotein increases, as do total cholesterol concentrations [25]. In patients with hypertension and/or insulin resistance, the metabolism of lipoproteins is delayed, and they remain in the blood circulation for a variety of reasons [26]. Cholesterol-rich and TG-rich apoB-containing remnant lipoproteins are taken up by macrophages, and cholesterol accumulates in atherosclerotic lesions [27, 28].

Non-HDL-C is easily calculated by subtracting HDL-C from total cholesterol. Non-HDL-C can provide a better risk estimation compared with LDL-C, in particular in hypertriglyceridemia combined with diabetes, metabolic syndrome, or chronic kidney disease. This is supported by a recent meta-analysis including 14 statin trials, 7 fibrate trials, and 6 nicotinic acid trials [29]. Non-HDL-C is used as an estimation of the total number of atherogenic particles in plasma (VLDL + intermediate-density lipoprotein $+\mathrm{LDL}$ ) and relates well to apoB levels. Non-HDL-C may be greatly affected by LDL-C and apoB concentrations [30]. A high correlation exists between the changes in non-HDL-C and TGs [31].

In our previous study, it was found that type 2 diabetes patients with hypertriglyceridemia can be divided into two groups: those with relatively low LDL-C and those with normal or high LDL-C. In the former group, TGs and sd-LDL were also higher than those in the latter group, but there was no difference in non-HDL$\mathrm{C}$ between the two groups $(4.9[4.0-5.4] \mathrm{mmol} / \mathrm{L}$ vs. 4.8 [4.5-5.2] mmol/L) [9]. Alternatively, the increase in TGs and decrease in LDL-C (calculated using Friedewald's formula) could have been synchronized [13, 32], and the change in non-HDL-C might have been offset. The non-HDL-C measurement formula evaluates the cholesterol levels of TGs and LDL, which are rich in apoB. An increase in sd-LDL reflecting hypertriglyceridemia and a decrease in buoyant LDL might occur simultaneously in type 2 diabetes patients. As evidence, this study showed that both the LDL-C and LDL fractions in PAG electrophoresis are negatively correlated with LDL-MI. If so, calculated non-HDL-C, regardless of the LDL-C assay 
method used, will largely reflect the decline in buoyant LDL present in some type 2 diabetes patients, and it is estimated that the increase in non-HDL-C would not be as expected. The changes in LDL composition would affect the measurement of non-HDL-C and thus impair its clinical reliability, and these changes might also affect the relationship between non-HDL-C and sd-LDL [9]. In contrast, another lipid marker that indirectly compares the changes in sd-LDL and buoyant LDL (i.e., the TG/LDL-C ratio) seems to more accurately reflect the pathophysiology of dyslipidemia in type 2 diabetes patients. A new subset of atherogenic lipoproteins consisting of LDL-C and TGs is proposed, with LDL-C and TGs serving as surrogates for $L D L /$ intermediate-density lipoprotein and VLDL, respectively. The TG/LDL-C ratio reflects $L D L$ and VLDL and can be a new predictive marker for sd-LDL increase. The fact that the cut-off point for the TG/LDL-C ratio was constant irrespective of statin treatment suggests that the TG/LDL-C ratio is universal and reliable for the prediction of sd-LDL increase.

The REDUCE-IT trial showed that treatment with icosapent ethyl significantly reduced CVD events without any change in non-HDL-C in patients with CVD risk and increased baseline TGs but well-controlled LDL-C $[31,33]$. The REDUCE-IT trial results may alter the approach to the management of hypertriglyceridemic patients whose lipid phenotype requires more intensive treatment beyond LDL-C lowering alone.

\section{Study strengths and limitations}

There are several strengths to this study. First, the reliable LDL-C direct assay, Metabolead LDL-C $\AA$ (Hitachi Kasei Diagnostic Systems), was used for LDL-C estimation, and the results were consistent with the lipoprotein PAG electrophoresis results [9]. Moreover, this direct method has already been shown to be consistent with ultracentrifugation, unless TGs exceed $11.3 \mathrm{mmol} / \mathrm{L}[15,16]$. There were no patients with a fasting TG level of $\geq 11.3 \mathrm{mmol} / \mathrm{L}$ in this study; therefore, any effect of hypertriglyceridemia on the LDL-C assay could be ruled out $[34,35]$. A study observing changes in LDL (measured by high-

performance liquid chromatography) before and after pemafibrate reported an increase in LDL, especially large, buoyant LDL, in addition to a decrease in sd-LDL after the treatment [36]. Conversely, it indicated that there was a relative reduction in baseline large, buoyant LDL in some patients with hypertriglyceridemia [13]. This is consistent with the decreases in baseline LDL-C (measured via the direct method) and baseline LDL fraction in PAG electrophoresis. Second, PAG electrophoresis, which is a simple and inexpensive method, was used for the estimation of sd-LDL and lipoprotein fractions.

This study also has several limitations. First, instead of directly measuring sd-LDL, determination of LDLMI by PAG electrophoresis was used as a substitute. However, many reports have indicated that the results of both are strongly correlated $[13,14]$. Second, there were relatively few target patients in this study. Statistically significant results were obtained despite the small number of patients in present study, however. Future studies should enroll a larger number of target patients. Third, the prevalence of CVD in the target patients in present study was low, and it was not possible to evaluate CVD due to quantitative changes in TG/LDL-C ratio or LDL-MI. Fourth, there may be some specificity in the clinical background of the patients included in present study. There was a high proportion of male patients. Patients treated with statins were older and had lower eGFR. It is generally known that Okinawan subjects (who were analyzed

Page 13/19 
in the present study) tend to develop obesity from an early age and that the frequencies of dyslipidemia and metabolic syndrome in these subjects are high $[37,38]$. It is therefore unclear whether the results of this study are universally applicable to all Japanese or other ethnic groups. Finally, this study did not show results for serum apolipoproteins such as apoB and apolipoprotein $\mathrm{E}$.

\section{Conclusion}

The TG/LDL-C ratio may offer a simple clinical tool for predicting increased sd-LDL. The TG/LDL-C ratio was useful in the pathologic evaluation of type 2 diabetes patients with high TGs compared with the recommended measurement of non-HDL-C. Even in statin-treated type 2 diabetes patients whose LDL-C remains within the therapeutic range, clear increases in TG/LDL-C ratio and sd-LDL with hypertriglyceridemia are inherent. In the ACCORD lipid study, combination therapy with a statin and fibrate could not be demonstrated to suppress CVD events in patients with type 2 diabetes [39]. However, in diabetes patients with high TGs and low HDL-C, the onset of CVD was persistently suppressed even after the ACCORD trial was completed [40]. A new large-scale clinical trial currently underway is evaluating the usefulness of TG-lowering therapy for suppressing the development of CVD [41]. The usefulness of the TG/LDL-C ratio for CVD prediction may also be demonstrated in that trial. It may therefore be necessary to review recommendations in the arteriosclerosis guidelines in the future [7].

\section{Declarations}

\section{Acknowledgements}

We would like to thank Dr. Akira Yamamoto for collecting patient data and FORTE Science Communications (www.forte-science.co.jp) for English language editing.

\section{Authors' contributions}

IK has full access to all of the data from the study and takes responsibility for the integrity of the data and the accuracy of the data analysis. GO and IK were involved in study design, interpreting the data, statistical analyses, creating tables and figures, and drafting the manuscript. ST was involved in study design. TW and YO were involved in interpreting the data and supervising the work. All authors have contributed significantly. All the authors have read the manuscript and approved this submission.

\section{Funding}

This research did not receive any specific grant from funding agencies in the public, commercial, or notfor-profit sectors.

\section{Availability of data and materials}

The collection of data that supports the findings in this study is available from Medical Plaza Daido Central. Data are available from the authors upon reasonable request and with permission of Medical 
Plaza Daido Central.

\section{Ethics approval and consent to participate}

In accordance with the Declaration of Helsinki, this retrospective study was approved by the Ethics Committee of Medical Plaza Daido Central (approval number 34-2020). Because only medical records were reviewed, patients enrolled in this study were exempted from signing the informed consent.

\section{Consent for publication}

Regarding publication of the research results, we explained that we could not reveal personal information and obtained verbal consent from all target patients.

\section{Competing interests}

The authors declare no conflict of interest.

\section{References}

1. Hoogeveen RC, Gaubatz JW, Sun W, Dodge RC, Crosby JR, Jiang J, et al. Small dense low-density lipoprotein-cholesterol concentrations predict risk for coronary heart disease: the Atherosclerosis Risk In Communities (ARIC) study. Arterioscler Thromb Vasc Biol. 2014;34:1069-77. doi:

10.1161/ATVBAHA.114.303284.

2. Chait A, Brazg RL, Tribble DL, Krauss RM. Susceptibility of small, dense, low-density lipoproteins to oxidative modification in subjects with the atherogenic lipoprotein phenotype, pattern B. Am J Med. 1993;94:350-6. doi: 10.1016/0002-9343(93)90144-e.

3. Sakai K, Koba S, Nakamura Y, Yokota Y, Tsunoda F, Shoji M, et al. Small dense low-density lipoprotein cholesterol is a promising biomarker for secondary prevention in older men with stable coronary artery disease. Geriatr Gerontol Int. 2018;18:965-72. doi: 10.1111/ggi.13287.

4. Hayashi T, Koba S, Ito Y, Hirano T. Method for estimating high sdLDL-C by measuring triglyceride and apolipoprotein B levels. Lipids Health Dis. 2017; doi: 10.1186/s12944-017-0417-6.

5. Noda H, Iso H, Irie F, Sairenchi T, Ohtaka E, Ohta H. Association between non-high-density lipoprotein cholesterol concentrations and mortality from coronary heart disease among Japanese men and women: the Ibaraki Prefectural Health Study. J Atheroscler Thromb. 2010;17:30-6. doi: 10.5551/jat.1016.

6. Manocha A, Bhargava S, Jain R, Kankra M, Singla P, Chugh P. Non-HDL as a valid surrogate marker of small dense LDL in a young Indian population. Indian J Clin Biochem. 2019 Jul;34(3):263-271. doi: 10.1007/s12291-018-0758-6.

7. Catapano AL, Graham I, De Backer G, Wiklund O, Chapman MJ, Drexel H, et al. 2016 ESC/EAS guidelines for the management of dyslipidaemias. Eur Heart J. 2016;37:2999-3058. doi: 10.1093/eurheartj/ehw272. 
8. Robinson JG, Wang S, Smith BJ, Jacobson TA. Meta-analysis of the relationship between non-highdensity lipoprotein cholesterol reduction and coronary heart disease risk. J Am Coll Cardiol. 2009;53:316-22. doi: 10.1016/j.jacc.2008.10.024.

9. Komiya I, Yamamoto A, Sunakawa S, Wakugami T. Pemafibrate decreases triglycerides and small, dense LDL, but increases LDL-C depending on baseline triglycerides and LDL-C in type 2 diabetes patients with hypertriglyceridemia: an observational study. Lipids Health Dis. 2021;20: 17. doi: 10.1186/s12944-021-01434-8. pmid: 33610176. doi: 10.1186/s12944-021-01434-8.

10. Ishibashi S, Yamashita S, Arai H, Araki E, Yokote K, Suganami H, et al. Effects of K-877, a novel selective PPARa modulator (SPPARMa), in dyslipidaemic patients: a randomized, double blind, active- and placebo-controlled, phase 2 trial. Atherosclerosis. 2016;249:36-43. doi: 10.1016/j.atherosclerosis.2016.02.029.

11. Fruchart JC. Pemafibrate (K-877), a novel selective peroxisome proliferator-activated receptor alpha modulator for management of atherogenic dyslipidaemia. Cardiovasc Diabetol. 2017; doi: 10.1186/s12933-017-0602-y.

12. Miller M, Cannon CP, Murphy SA, Qin J, Ray KK, Braunwald E. Impact of triglyceride levels beyond low-density lipoprotein cholesterol after acute coronary syndrome in the PROVE IT-TIMI 22 trial. J Am Coll Cardiol. 2008;51:724-30. doi: 10.1016/j.jacc.2007.10.038.

13. Tani S, Matsumoto M, Nagao K, Hirayama A. Association of triglyceride-rich lipoproteins-related markers and low-density lipoprotein heterogeneity with cardiovascular risk: effectiveness of polyacrylamide-gel electrophoresis as a method of determining low-density lipoprotein particle size. J Cardiol. 2014;63:60-8. doi: 10.1016/j.jjcc.2013.06.018.

14. Imajo $\mathrm{K}$, Hyogo $\mathrm{H}$, Yoneda $\mathrm{M}$, Honda $\mathrm{Y}$, Kessoku T, Tomeno W, et al. LDL-migration index (LDL-MI), an indicator of small dense low-density lipoprotein (sdLDL), is higher in non-alcoholic steatohepatitis than in non-alcoholic fatty liver: a multicenter cross-sectional study. PLoS One. 2014; doi: 10.1371/journal.pone.0115403.

15. Miida T, Nishimura K, Hirayama S, Miyamoto Y, Nakamura M, Masuda D, et al. Homogeneous assays for LDL-C and HDL-C are reliable in both the postprandial and fasting state. J Atheroscler Thromb. 2017;24:583-99. doi: 10.5551/jat.40006.

16. Miller WG, Myers GL, Sakurabayashi I, Bachmann LM, Caudill SP, Dziekonski A, et al. Seven direct methods for measuring HDL and LDL cholesterol compared with ultracentrifugation reference measurement procedures. Clin Chem. 2010 Jun;56(6):977-86. doi: 10.1373/clinchem.2009.142810.

17. Kanda Y. Investigation of the freely available easy-to-use software 'EZR' for medical statistics. Bone Marrow Transplant 2013; 48: 452-458. doi: 10.1038/bmt.2012.244.

18. Yoshida A, Kouwaki M, Matsutani Y, Fukuchi Y, Naito M. Usefulness of serum total cholesterol/triglyceride ratio for predicting the presence of small, dense LDL. J Atheroscler Thromb. 2004;11:215-9. doi: 10.5551/jat.11.215.

19. Moriyama K, Takahashi E. Non-HDL cholesterol is a more superior predictor of small-dense LDL cholesterol than LDL cholesterol in Japanese subjects with TG levels $<400 \mathrm{mg} / \mathrm{dL}$. J Atheroscler 
Thromb. 2016;23:1126-1137. doi: 10.5551/jat.33985.

20. Hiramatsu K, Yamada T, Yukimura Y, Komiya I, Ichikawa K, Ishihara M, et al. A screening test to identify aldosterone-producing adenoma by measuring plasma renin activity. Results in hypertensive patients. Arch Intern Med. 1981; 141(12):1589-93.

21. Kastelein JJP, van der Steeg WA, Holme I, Gaffney M, Cater NB, Barteret P, et al. Lipids, apolipoproteins, and their ratios in relation to cardiovascular events with statin treatment. Circulation. 2008;117:3002-3009. doi: 10.1161/CIRCULATIONAHA.107.713438.

22. You J, Wang Z, Lu G, Chen Z. Association between the non-high-density lipoprotein cholesterol to high-density lipoprotein cholesterol ratio and the risk of coronary artery disease. Biomed Res Int. 2020 Mar 7;2020:7146028. doi: 10.1155/2020/7146028.

23. McLaughlin T, Abbasi F, Cheal K, Chu J, Lamendola C, Reaven G. Use of metabolic markers to identify overweight individuals who are insulin resistant. Ann Intern Med 2003;139:802-809. doi: 10.7326/0003-4819-139-10-200311180-00007.

24. Pischon T, Girman CJ, Sacks FM, Rifai N, Stampfer MJ, Rimm EB. Non-high-density lipoprotein cholesterol and apolipoprotein $B$ in the prediction of coronary heart disease in men. Circulation. 2005;112:3375-83. doi: 10.1161/CIRCULATIONAHA.104.532499.

25. Chapman MJ, Ginsberg HN, Amarenco P, Andreotti F, Borén J, Catapano AL, et al. Triglyceride-rich lipoproteins and high-density lipoprotein cholesterol in patients at high risk of cardiovascular disease: evidence and guidance for management. Eur Heart J. 2011;32:1345-61. doi: 10.1093/eurheartj/ehr112.

26. Sørensen LP, Andersen IR, Søndergaard E, Gormsen LC, Schmitz O, Christiansen JS, et al. Basal and insulin mediated VLDL-triglyceride kinetics in type 2 diabetic men. Diabetes. 2011;60:88-96. doi: $10.2337 / \mathrm{db} 10-0564$.

27. Joshi PH, Khokhar AA, Massaro JM, Lirette ST, Griswold ME, Martin SS, et al. Remnant lipoprotein cholesterol and incident coronary heart disease: the Jackson Heart and Framingham Offspring Cohort Studies. J Am Heart Assoc. 2016; doi: 10.1161/JAHA.115.002765.

28. Borén J, Williams KJ. The central role of arterial retention of cholesterol-rich apolipoprotein-Bcontaining lipoproteins in the pathogenesis of atherosclerosis: a triumph of simplicity. Curr Opin Lipidol. 2016;27:473-83. doi: 10.1097/MOL.0000000000000330.

29. Silverman MG, Ference BA, Im K, Wiviott SD, Giugliano RP, Grundy SM, et al. Association between lowering LDL-C and cardiovascular risk reduction among different therapeutic interventions: a systematic review and meta-analysis. JAMA. 2016;316:1289-97. doi: 10.1001/jama.2016.13985.

30. Ference BA, Kastelein JJP, Ray KK, Ginsberg HN, Chapman MJ, Packard CJ, et al. Association of triglyceride-lowering LPL variants and LDL-C-lowering LDLR variants with risk of coronary heart Disease. JAMA. 2019;321:364-73. doi: 10.1001/jama.2018.20045.

31. Marston NA, Giugliano RP, Im K, Silverman MG, O'Donoghue ML, Wiviott SD, et al. Association between triglyceride lowering and reduction of cardiovascular risk across multiple lipid-lowering 
therapeutic classes: a systematic review and meta-regression analysis of randomized controlled trials. Circulation. 2019;140:1308-1317. doi: 10.1161/CIRCULATIONAHA.119.041998.

32. Hirose T, Teramoto T, Abe K, Taneyama T, J-BENEFIT study group. Determinants of bezafibrateinduced improvements in LDL cholesterol in dyslipidemic patients with diabetes. J Atheroscler Thromb. 2015;22:676-84. doi: 10.5551/jat.27425.

33. Boden WE, Bhatt DL, Toth PP, Ray KK, Chapman MJ, Lüscher TF. Profound reductions in first and total cardiovascular events with icosapent ethyl in the REDUCE-IT trial: why these results usher in a new era in dyslipidaemia therapeutics. Eur Heart J. 2020;41:2304-2312. doi:

10.1093/eurheartj/ehz778.

34. Langlois MR, Descamps OS, van der Laarse A, Weykamp C, Baum H, Pulkki K, et al. Clinical impact of direct HDLC and LDLc method bias in hypertriglyceridemia. a simulation study of the EAS-EFLM Collaborative Project Group. Atherosclerosis. 2014;233:83-90. doi:

10.1016/j.atherosclerosis.2013.12.016.

35. Miida T, Nishimura $K$, Okamura T, Hirayama $S$, Ohmura $H$, Yoshida $H$, et al. A multicenter study on the precision and accuracy of homogeneous assays for LDL-cholesterol: comparison with a betaquantification method using fresh serum obtained from non-diseased and diseased subjects. Atherosclerosis. 2012;225:208-15. doi: 10.1016/j.atherosclerosis.2012.08.022.

36. Yamashita S, Arai H, Yokote K, Araki E, Matsushita M, Nojima T, et al Efficacy and safety of pemafibrate, a novel selective peroxisome proliferator-activated receptor a modulator (SPPARMa): pooled analysis of phase 2 and 3 studies in dyslipidemic patients with or without statin combination. Int J Mol Sci. 2019;20:5537. doi: 10.3390/ijms20225537.

37. Asato Y, Katsuren K, Ohshiro T, Kikawa K, Shimabukuro T, Ohta T. Relationship between lipid abnormalities and insulin resistance in Japanese school children. Arterioscler Thromb Vasc Biol. 2006;26:2781-6. doi: 10.1161/01.ATV.0000245804.56871.31.

38. Mimura G, Nakamasu J, Irie M. Incidence of hyperlipemia in diabetics in Okinawa and its relation to ischemic heart disease. Tohoku J Exp Med. 1983;141 Suppl:611-7. doi:

10.1620/tjem.141.suppl_611.

39. ACCORD Study Group, Ginsberg HN, Elam MB, Lovato LC, Crouse III JR, Leiter LA, Linz P, et al. Effects of combination lipid therapy in type 2 diabetes mellitus. N Engl J Med 2010;362:1563-1574. doi: 10.1056/NEJMoa1001282.

40. Zhu L, Hayen A, Bell KJL. Legacy effect of fibrate add-on therapy in diabetic patients with dyslipidemia: a secondary analysis of the ACCORDION study. Cardiovasc Diabetol. 2020;19:28. doi: 10.1186/s12933-020-01002-x.

41. Pradhan AD, Paynter NP, Everett BM, Glynn RJ, Amarenco P, Elam M, et al. Rationale and design of the pemafibrate to reduce cardiovascular outcomes by reducing triglycerides in patients with diabetes (PROMINENT) study. Am Heart J. 2018;206:80-93. doi: 10.1016/j.ahj.2018.09.011.

\section{Figures}


Fig. $1 \mathrm{~A}$



Fig. 1B

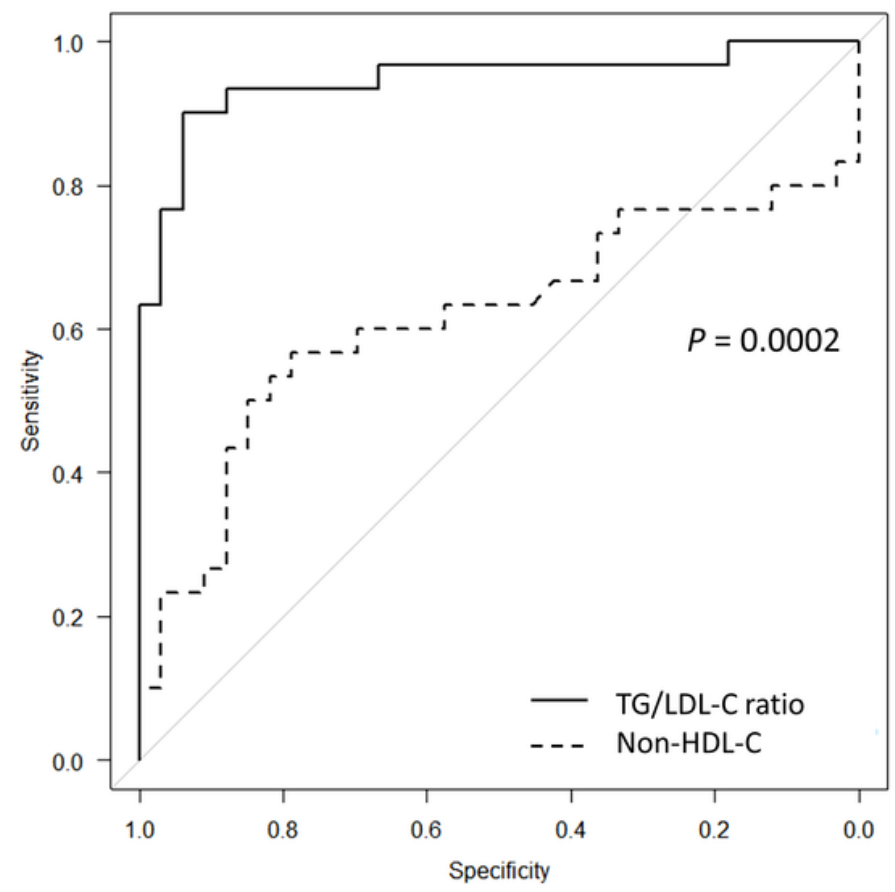

\section{Figure 1}

ROC curves for TG/LDL-C ratio and non-HDL-C for predicting high LDL-MI ( $\geq 0.400$ ) among all patients (A) and those not treated with statins (B) AUCs of TG/LDL-C ratio (solid line) and non-HDL-C (dotted line) among all patients (A) and those not treated with statins (B). The AUC of the TG/LDL-C ratio was significantly greater than that of non-HDL-C among all patients $(P=0.0035, X 2$ test), and those not treated with statins $(P=0.0002)$. ROC, receiver operator characteristic; LDL-MI, LDL migration index; AUC, area under the curve; TG, triglyceride; LDL-C, low-density-lipoprotein cholesterol; non-HDL-C, non-highdensity-lipoprotein cholesterol. 